\title{
Kein Grundrechtsanspruch auf «Homeschooling»?
}

Bemerkungen zum Urteil des Bundesgerichts 2C_1005/2018 vom 22. August 2019 (zur Publikation vorgesehen)

Mathias Kaufmann *

In ihrem zur amtlichen Veröffentlichung vorgesehenen Urteil 2C_1005/2018 vom 22. August 2019 hat die II. öffentlichrechtliche Abteilung des Bundesgerichts erstmals erwogen, dass es keinen auf Art. 13 Abs. 1 der Bundesverfassung fussenden Grundrechtsanspruch auf Heimunterricht (sog. «Homeschooling») gibt. Der vorliegende Beitrag fasst das Urteil zusammen und äussert sich zur Stichhaltigkeit der in ihm präsentierten höchstrichterlichen Argumentation.

I. Sachverhalt 336

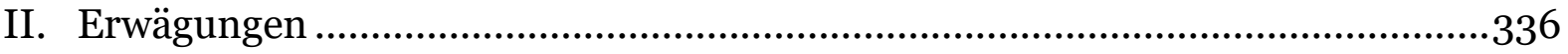

III. Bemerkungen .................................................................................................. 337

Zitiervorschlag: Mathias Kaufmann, Kein Grundrechtsanspruch auf «Homeschooling»?, in: sui-generis 2019, S. 335

URL: $\quad$ sui-generis.ch/113

DOI: $\quad$ https://doi.org/10.21257/sg.113

* Dr. iur. Mathias Kaufmann (mathias.kaufmann@gmail.com), Leiter des Rechtsdiensts der Pädagogischen Hochschule Bern, Lehrbeauftragter an den Universitäten Zürich und Freiburg. 


\section{Sachverhalt}

1 Am 2. Juni 2017 ersuchte die Beschwerdeführerin das Erziehungsdepartement des Kantons Basel-Stadt darum, ihren Sohn (geboren 2009) während des Schuljahrs 2017/18 zuhause unterrichten (lassen) zu dürfen. Mit Verfügung vom 10. August 2017 lehnte die Volksschulleitung das Gesuch ab, woraufhin die Beschwerdeführerin zuerst erfolglos ans Erziehungsdepartement und hernach mit Rekurs ans Verwaltungsgericht gelangte. Mit Urteil vom 25. September 2018 wies das Appellationsgericht des Kantons Basel-Stadt als Verwaltungsgericht den Rekurs ab, was die Beschwerdeführerin dazu bewog, beim Bundesgericht mit dem Antrag Beschwerde zu erheben, das Urteil des Appellationsgerichts aufzuheben und ihrem Sohn für das Schuljahr 2017/18 privaten Heimunterricht zu bewilligen.

2 Begründet wurde die Notwendigkeit des Heimunterrichts seitens der Beschwerdeführerin mit den negativen Erfahrungen, welche sie und ihr Sohn in den ersten beiden Schuljahren (2015/16 und 2016/17) gemacht hätten. Obschon ihr Sohn hochbegabt und im zweiten Schuljahr gemobbt worden sei, habe die Schule seiner Hochbegabung nicht genug Rechnung getragen und gegen das Mobbing nicht genug unternommen. ${ }^{1}$

\section{Erwägungen}

3 Aufgrund der Tatsache, dass das Schuljahr 2017/18, für welches die Beschwerdeführerin «Homeschooling» beantragt hatte, im Entscheidzeitpunkt schon

\footnotetext{
Vgl. Urteil des Appellationsgerichts BS (als Verwaltungsgericht) VD.2018.97 vom 25. September 2018, E. 5 .
}

(längst) vorbei war, galt es vor Bundesgericht - wie bereits vor der Vorinstanz -2 zunächst, auf die Prozessvoraussetzung der materiellen Beschwer (vgl. Art. 89 Abs. 1 lit. b und c BGG) einzugehen. Das Bundesgericht entschied hierzu, dass sich die von der Beschwerdeführerin aufgeworfene Frage nach der Vereinbarkeit von $\S 135$ des basel-städtischen Schulgesetzes vom 4. April 1929 (SchulG; SG 410.100) mit dem höherrangigen Recht ohne weiteres wieder stellen könnte, ohne dass eine rechtzeitige Überprüfung möglich wäre, und dass eine Klärung im öffentlichen Interesse liegt, weshalb auf das Erfordernis des aktuellpraktischen Rechtsschutzinteresses nach Art. 89 Abs. 1 lit. c BGG ausnahmsweise $\mathrm{zu}$ verzichten und auf die form- und fristgerecht eingereichte Beschwerde in öffentlichrechtlichen Angelegenheiten einzutreten sei (E. 1).

4 In materieller Hinsicht prüfte die II. öffentlichrechtliche Abteilung des Bundesgerichts im Rahmen einer inzidenten Normenkontrolle sodann, ob sich Art. 19 (i. V. m. Art. 62 Abs. 2) und/oder Art. 13 Abs. 1 BV ein Anspruch auf «Homeschooling» entnehmen lässt (vgl. die E. 4 und 5). Mit Blick auf das in Art. 19 BV verankerte Recht auf ausreichenden Grundschulunterricht verneinte sie dies unter (knappem) Hinweis auf ihre eigene Praxis, zwei Lehrmeinungen und die vorinstanzlichen Ausführungen

2 Vgl. Urteil des Appellationsgerichts BS (als Verwaltungsgericht) VD.2018.97 vom 25. September 2018, E. 1.2.1-1.2.3. 
(E. 4.3).3 In Bezug auf das als Teilgehalt von Art. 13 Abs. 1 BV (und Art. 8 EMRK) anerkannte elterliche Erziehungsrecht fiel die Prüfung etwas ausführlicher aus, zumal das Bundesgericht bisher noch keine Gelegenheit hatte, zur Frage nach einem allfälligen auf demselben beruhenden Anspruch auf «Homeschooling» Stellung zu nehmen (vgl. die E. 5.1): Nach vertiefter Auseinandersetzung mit dem besagten Erziehungsrecht, namentlich der einschlägigen Rechtsprechung des EGMR (vgl. die E. 5.2-5.4), kam die II. öffentlichrechtliche Abteilung in der E. 5.5 indessen zum Ergebnis, dass auch aus Art. 8 EMRK (i. V. m. Art. 2 des ersten Zusatzprotokolls zur EMRK) kein Recht auf häusliche Privatbeschulung fliesse und dass derzeit - entgegen einer «vereinzelten Lehrmeinung»4 - keine Veranlassung dazu gegeben sei, ein solches Recht gestützt auf Art. 13 Abs. 1 BV zu bejahen; ob bzw. inwieweit «Homeschooling» zulässig ist, liege im Ermessen der Kantone.

5 Zu klären blieb damit einzig noch, ob das «Homeschooling»-Gesuch der Beschwerdeführerin in Anwendung von $§ 135$ SchulG/BS seinerzeit abgelehnt werden durfte (vgl. die E.6.1-6.3), wobei das Bundesgericht dies - vor dem Hintergrund des seiner Ansicht nach fehlenden verfassungsmässigen Anspruchs auf

$3 \longdiv { \text { Vgl. Urteil des Bundesgerichts 2C_738/2010 vom } }$ 24. Mai 2011, E. 3.3.2, Ulrich Häfelin/Walter Haller/Helen Keller/Daniela Thurnherr, Schweizerisches Bundesstaatsrecht, 9. Aufl., Zürich/ Basel/Genf 2016, N. 925e, René Rhinow/Markus Schefer/Peter Uebersax, Schweizerisches Verfassungsrecht, 3. Aufl., Basel 2016, N. 3480a, sowie Urteil des Appellationsgerichts BS (als Verwaltungsgericht) VD.2018.97 vom 25. September 2018, E. 3.2.

4 Johannes Reich, «Homeschooling» zwischen elterlichem Erziehungsrecht, staatlicher Schulpflicht und Kindeswohl, ZBl 2012567 ff., S. 598 f.
«Homeschooling» - lediglich auf Willkür hin prüfen musste (vgl. die E. 2.1 und 6). Es hielt fest, die Vorinstanz habe willkürfrei erwogen, dass das Vorliegen eines besonderen Grunds i. S.v. §135 Abs. 2 lit. a SchulG/BS nicht erstellt sei (E. 6.3),5 und wies die Beschwerde ab (E. 7).

\section{Bemerkungen}

6 Vergleicht man vorab die Argumentation des Bundesgerichts mit derjenigen der Vorinstanz, fällt auf, dass die II. öffentlichrechtliche Abteilung in ihrem Entscheid in einem ganz wesentlichen Punkt eine methodische Korrektur vornahm: Während das basel-städtische Appellationsgericht - der «vereinzelten Lehrmeinung» von Reich ${ }^{6}$ folgend - von einem durch $\S 135$ SchulG/BS bewirkten Eingriff in den Schutzbereich des durch Art. 13 Abs. 1 BV und Art. 8 EMRK gewährleisteten elterlichen Erziehungsrechts ausging und folgerichtig zur «BV 36-Prüfung» schritt,7 erachtete das Bundesgericht das Recht auf Privat- und Familienleben als durch $\S 135$ SchulG/BS nicht tangiert (vgl. vor allem die E. 5.5). ${ }^{8}$

$5 \longdiv { \text { Vgl. Urteil des Appellationsgerichts BS (als Ver- } }$ waltunsgericht) VD.2018.97 vom 25. September 2018, E. 5.4.

6 Vgl. Reich, «Homeschooling» (Fn. 4), S. 599: «[E]ine kantonale Vorschrift, wonach die Schulpflicht nur durch Schulbesuch erfüllt werden kann [...], [greift] dann in die verfassungsrechtlich geschützten Erziehungsrechte der Eltern [ein], wenn der elterlich erteilte häusliche Privatunterricht im konkreten Fall sowohl den Anforderungen von Art. 62 Abs. 2 Satz 2 BV genügt als auch das Wohl des Kindes wahrt.»

7 Vgl. Urteil des Appellationsgerichts BS (als Verwaltungsgericht) VD.2018.97 vom 25. September 2018, E. 4 .

8 Umgekehrt verlief die Prozessgeschichte etwa im «Kindergarten-Yoga-Fall»: Das Zürcher Verwaltungsgericht hatte die Betroffenheit des Schutzbereichs von Art. 15 BV verneint, woraufhin das Bundesgericht konstatierte, die Religionsfreiheit sei zwar (geringfügig) tangiert, nicht aber ver- 
Damit konnte es das Bundesgericht dann im Wesentlichen sein Bewenden haben lassen - und sich in der E. 6, wie gesehen, auf eine Willkürkontrolle beschränken.

7 Um es vorwegzunehmen: Der II. öffentlichrechtlichen Abteilung des Bundesgerichts ist mit Blick auf das vorliegend interessierende Leiturteil m. E. bloss insoweit zuzustimmen, als sie zum Ergebnis gelangt, dass weder Art. 19 (i. V. m. Art. 62 Abs. 2) BV noch Art. 13 Abs. 1 BV und Art. 8 EMRK der Beschwerdeführerin ein verfassungsmässiges Recht auf privaten Heimunterricht für ihren Sohn im Schuljahr 2017/18 verschaffte. Im Übrigen ist, wie mir scheint, Kritik angezeigt, und zwar insbesondere daran, dass sich das Bundesgericht im Entscheid 2C_1005/2018 mit (fast) keinem Wort zur Tragweite des zwar nicht als verletzt gerügten (vgl. Art. 106 Abs. 2 BGG), aber dennoch in hohem Mass entscheidrelevanten Art. 11 $B V$ äusserte. Erstens ging es - wegen des dahingefallenen Rechtsschutzinteresses - ohnehin nicht mehr um den konkreten Einzelfall (vgl. die E. 1.2); zweitens (und vor allem) umfasst der Individualschutzbereich von Art. 11 Abs. 1 BV nach der hier vertretenen Auffassung den gesuchten grundrechtlich geschützten «Homeschooling»-Anspruch. Falls also das Bundesgericht im Urteil 2C_1005/2018 eigentlich sagen wollte, dass es (derzeit) überhaupt kein subjektives Verfassungsrecht auf Heimunterricht gibt, wäre eine

letzt; begründet wurde beides damit, dass dem streitgegenständlichen «Yoga-Unterricht» an einem öffentlichen Kindergarten die Bekenntnishaftigkeit abging (vgl. Urteil des Verwaltungsgerichts Zürich VB.2012.00168 vom 11. Juli 2012, E. 3.4, 4; Urteil des Bundesgerichts 2C_897/2012 vom 14. Februar 2013, E. 4.2 f.). entsprechende Klarstellung - wenn auch, wie sogleich näher darzulegen sein wird, unzutreffend - hilfreich gewesen. Falls es dies hingegen nicht sagen wollte, d. h. die Möglichkeit eines Grundrechtsanspruchs auf «Homeschooling» nach wie vor in Betracht zieht, hätte es das Recht von Amtes wegen anwenden (vgl. Art. 106 Abs. 1 BGG) und eine Art. 11 BV berücksichtigende Normenkontrolle vornehmen sollen. Wäre es dabei zum Schluss gekommen, dass der besagte Anspruch (doch) existiert, hätte dies freilich eine «freie» Einzelaktkontrolle nötig gemacht.

8 Das Bundesgericht hat sich bisher beharrlich geweigert, Art. 11 (Abs. 1) BV «positiv» zu konkretisieren - und dies mit Zustimmung (oder jedenfalls ohne grosse Gegenwehr) aus dem Schrifttum. ${ }^{9}$

9 Vgl. aus der höchstrichterlichen Praxis BGE 126 II 377 E. 5; BGE 129 I 12 E. 10.5.3; BGE 131 V 9 E. 3.5.1.2; BGE 135 I 153 E. 2.2.2; Urteile des Bundesgerichts 2P.324/2001 vom 28. März 2002, E. 4.2; 1B_122/2010 vom 13. August 2010, E. 3.2.3; 2C_832/2010 vom 2. Februar 2011, E. 2.2.2; aus der Literatur Eva Maria Belser/ Bernhard Waldmann, Grundrechte II, Zürich/ Basel/Genf 2012, Kap. 2 N. 112, 116; Giovanni Biaggini, Kommentar zur Bundesverfassung, 2. Aufl., Zürich 2017, Art. 11 N. 4 f.; Astrid Epiney/ Bernhard Waldmann, Soziale Grundrechte und soziale Zielsetzungen, in: Detlef Merten/HansJürgen Papier (Hrsg.), Handbuch der Grundrechte VII/2, Heidelberg/Zürich/St. Gallen 2007, $\S 224, \quad$ N. 49; Häfelin/Haller/Keller/Thurnherr (Fn. 3), N. 291; Axel Tschentscher/Andreas Lienhard/Franziska Sprecher, Öffentliches Recht, 2. Aufl., Zürich/St. Gallen 2019, N. 259; Ruth Reusser/Kurt Lüscher, in: Bernhard Ehrenzeller/ Benjamin Schindler/Rainer J. Schweizer/Klaus A. Vallender (Hrsg.), St. Galler Kommentar zur Bundesverfassung, 3. Aufl., Zürich/Basel/Genf/ St. Gallen, Art. 11 N. 30 ff. Explizit bejaht wird die Justiziabilität von Art. 11 Abs. 1 BV von Regina Kiener/Walter Kälin/Judith Wyttenbach, Grundrechte, 3. Aufl., Bern 2018, § 37 N. 9, 20, und Axel Tschentscher, in: Bernhard Waldmann/Eva Maria Belser/Astrid Epiney (Hrsg.), Basler Kommentar zur Bundesverfassung, Basel 2015, Art. 11 N. 11; vgl. auch Christine Kaufmann, in: Giovanni Biaggini/Thomas Gächter/Regina Kiener 
Im ersten zum damals noch völlig neuen Grundrecht der Kinder und Jugendlichen auf besonderen Integritätsschutz und auf Entwicklungsförderung ergangenen Leitentscheid vom September 2000 stellte die II. öffentlichrechtliche Abteilung des Bundesgerichts fest, es sei «fraglich», ob Art. $11 \mathrm{Abs} .1 \mathrm{BV}$ in seiner Ausprägung als Schutzanspruch neben der persönlichen Freiheit eine «weitergehende Tragweite» zukommt; um aus ihm einen Anspruch auf eine Aufenthaltsbewilligung abzuleiten, erweise sich dieses Grundrecht jedenfalls als «zu unbestimmt». Im Übrigen werde mit dem in Art. 11 Abs. 1 BV verankerten Förderungsanspruch einzig der Gesetzgeber in die Pflicht genommen, weshalb jenem lediglich programmatischer Charakter eigne sowie «keine zusätzlichen klagbaren subjektiven Rechte» zu entnehmen seien. ${ }^{10}$ - Einmal abgesehen davon, dass sich aus (sehr) unbestimmt abgefassten Verfassungs- bzw. Völkerrechtsnormen durchaus Ansprüche auf fremdenpolizeiliche Bewilligungen ergeben können, ${ }^{11}$ verlieh diese

(Hrsg.), Staatsrecht, 2. Aufl., Zürich/St. Gallen 2015, § 41 N. 52 f.; Jörg Paul Müller/Markus Schefer, Grundrechte in der Schweiz, 4. Aufl., Bern 2008, S. 807; Johannes Reich, «Schutz der Kinder und Jugendlichen» als rechtsnormatives und expressives Verfassungsrecht, ZSR 2012 I 363 ff., S. 385 f.; Rhinow/Schefer/Uebersax (Fn. 3), N. 1340 ff. Vgl. zur Genese von (und «Motivation» hinter) Art. 11 BV Reich, "Homeschooling» (Fn. 4), S. 365 ff., 373 f., 374 f.

10 BGE 126 II 377 E. 5d. Biaggini (Fn. 9), Art. 11 N. 4, bezeichnet den Förderungsanspruch gemäss Art. 11 Abs. 1 BV unter Verweisung auf dieses Urteil (und BGE 143 I 21) als «sozialzielähnlich».

11 Angesprochen ist Art. 8 EMRK; das Bundesgericht betont zwar regelmässig, dass die EMRK «praxisgemäss keinen Anspruch auf Einreise und Aufenthalt» verschaffe, nur um dann jedoch fortzufahren, dass es Art. 8 EMRK «verletzen» könne, «wenn einer ausländischen Person [...] die Anwesenheit untersagt [...] wird» (BGE 142 II 35 E. 6.1; BGE 139 I 330 E. 2.1; Urteil des Bundesgerichts 2C_786/2018 vom 27. Mai 2019, E. 3.1, 3.2.1). Vgl. hierzu ferner BGE 144 I 266 E. 3 f., wo höchstrichterliche Erwägung Art. 11 BV den (unberechtigten) Ruf, nicht justiziabel bzw. kein eigenständiges Grundrecht $\mathrm{zu}$ sein. ${ }^{12}$ Dabei ist es ein intendiertes Wesensmerkmal sämtlicher Grundrechtssätze, (entwicklungs-)offen formuliert zu sein, und ist es die wohl wichtigste Aufgabe des Bundesgerichts als Verfassungsgericht, den seitens des Bundesund der kantonalen Verfassungsgeber in die Grundrechtskataloge aufgenommenen Individualrechten in schöpferischer Verfassungsrechtsprechung Konturen zu verleihen - und zwar allen.13

9 Ein Grundrecht zu konkretisieren heisst, seinen (Individual-)Schutzbereich abzustecken, was wiederum gleichbedeutend ist mit dem Sichtbarmachen der in ihm enthaltenen spezifischen Anspruchspositionen der jeweiligen Grundrechtsträgerinnen und -träger gegenüber dem Staat

sich auch folgende spannende (und verallgemeinerungsfähige) Aussage findet: «Die Frage, $o b$ eine Wegweisungsmassnahme im Einzelfall in den Schutzbereich des Privatlebens fällt, ist mit der konkreten Interessenabwägung [= Verletzungsfrage] derart verwoben, dass eine Abgrenzung künstlich erscheint»; Christoph Grabenwarter/Katharina Pabel, Europäische Menschenrechtskonvention, 6. Aufl., München/Basel/Wien 2016, § 22 N. 72 ff.; mit Blick auf Art. 13 Abs. 1 BV etwa Biaggini (Fn. 9), Art. 13 N. 7.

12 So Biaggini (Fn. 9), Art. 11 N. 4 («nicht um ein eigenständiges Grundrecht»), 5 («[e]igenständig einklagbare Rechtspositionen werden durch BV 11 Abs. 1 nicht begründet»); Urteil des Bundesgerichts 2C_832/2010 vom 2. Februar 2011, E. 2.2.2 («kein verfassungsmässiges Recht»). Vgl. zur (Norm-)Justiziabilität als Kriterium des Vorliegens verfassungsmässiger Rechte Mathias Kaufmann, Der öffentlichrechtliche Anspruch, Diss. Zürich, Zürich/Basel/Genf 2015, S. 51 ff., $356 \mathrm{f}$.

13 Vgl. Kaufmann (Fn. 12), S. 90-92; Müller/Schefer (Fn. 9), S. 812 f. Gemäss Belser/Waldmann (Fn. 9), Kap. 2 N. 116, hat Art. 11 Abs. 1 BV «bis anhin noch nicht die Konturen erlangt [...], die für eine gerichtliche Durchsetzung erforderlich sind». Dass es just die Gerichte sind, die Art. 11 BV im Rahmen seiner Durchsetzung zu konturieren haben, ging hier vergessen. 
(und allenfalls Privaten; vgl. Art. 35 Abs. 3 BV). Dieses Sichtbarmachen obliegt nicht allein der Verfassungsjustiz, sondern auch dem Gesetzgeber und der Verwaltung (vgl. Art. 35 Abs. 1 und 2 BV), wiewohl gesetzliche und behördliche Grundrechtskonkretisierungen häufig in der Form von Grundrechtseinschränkungen i. S. v. Art. 36 BV daherkommen - und noch häufiger als solche empfunden werden. ${ }^{14}$

Mit dem Schutzbereich des Anspruchs von Kindern und Jugendlichen auf besonderen Schutz zum ersten Mal näher auseinandergesetzt hat sich das Bundesgericht in BGE 144 II 233, und zwar mit dem Resultat, dass die «LOVE LIFE - bereue nichts»-Präventionskampagne des BAG gegen HIV und andere Geschlechtskrankheiten diesen nicht tangiere (vgl. die E. 8), weshalb die Voraussetzung des Berührtseins der Beschwerdeführenden in ihren Rechten gemäss Art. 25a Abs. 1 VwVG nicht erfüllt sei und gegen die besagte Kampagne kein Rechtsschutz erlangt werden könne. Klargestellt hat das Bundesgericht damit, dass Kinder nicht nur keinen aus Art. 11 BV fliessenden verfassungsrechtlichen Anspruch darauf haben, von der «LOVE LIFE»-Kampagne verschont $\mathrm{zu}$ bleiben, sondern dass dieselbe nicht einmal einen Eingriff in den Schutzbereich von Art. 11 BV bewirkt.

Dass das Bundesgericht in BGE 144 II 233 faktisch eine Zumutbarkeitsprüfung vornahm und die Beschwerdeführenden

14 Vgl. zur traditionellen «Eingriffs-» oder «Schranken-» sowie zur (von Georg Müller und Marcel Bolz geprägten) «Konkretisierungstheorie» der Grundrechte Alain Griffel, Bauen im Spannungsfeld zwischen Eigentumsgarantie und Bauvorschriften, ZBl 2002169 ff., S. 171 ff. m. H. auf die genannten Autoren. faktisch durchaus Rechtsschutz erhielten, sei hier nur am Rand erwähnt. Interessant ist in vorliegendem Zusammenhang vielmehr, dass das Bundesgericht bei der Eruierung des Geltungsbereichs des verfassungsrechtlichen Anspruchs auf Kindesschutz in Bezug auf staatliche Informationskampagnen mit sexuellem Inhalt auf das strafbewehrte Verbot abgestellt hat, Personen unter 16 Jahren pornografische Aufnahmen und Abbildungen zu zeigen (vgl. Art. 197 Abs. 1 StGB), und dasselbe ausdrücklich als «Konkretisierung von Art. 11 BV» bezeichnete (vgl. die E. 8.2.2 f.). Insofern, als es e contrario strafrechtlich - und demnach laut Bundesgericht auch verfassungsrechtlich - zulässig ist, Kinder im öffentlichen Raum mit bloss «sexualisierten» Darstellungen zu konfrontieren, könnte man Art. 197 Abs. 1 StGB allerdings zugleich eine (zumindest leicht) grundrechtseinschränkende Wirkung zusprechen, woraus erhellt, dass für die «LOVE LIFE»-Kampagne letztlich dasselbe gilt: Wäre diese pornografischer Natur, würde sie Art. 11 Abs. 1 BV nämlich verletzen; hätte das BAG auf die strittigen «Sexszenen» verzichtet, läge dagegen gar keine Grundrechtseinschränkung vor.

12 (Zwischen-)Fazit: Weil es der herrschenden Dogmatik zufolge zwischen der Nichttangierung und der Verletzung eines Grundrechts zwingend Raum für gerechtfertigte Einschränkungen geben muss, stellt die in BGE 144 II 233 beurteilte Präventionskampagne eine zulässige Verkürzung des durch Art. 11 BV gewährleisteten Schutzanspruchs dar. Das Bundesgericht hätte die betreffende Beschwerde gutheissen (und aus Gründen der Prozessökonomie gleich selber die 
Rechtmässigkeit der Kampagne feststellen) müssen. ${ }^{15}$

Mit Blick auf den vom Bundesgericht im Urteil 2C_1005/2018 (mehr oder weniger umfassend) verneinten verfassungsmässigen Anspruch auf Heimunterricht ist die Ausgangslage ähnlich. Im Raum stand hier allerdings nicht die «Grundrechtsirrelevanz» eines Verwaltungsrealakts, sondern primär diejenige einer Gesetzesbestimmung, genauer: der in $\S 135$ Abs. 2 SchulG/BS aufgestellten Voraussetzungen des Erhalts einer Bewilligung für die Erteilung von «Privatunterricht». Festzuhalten ist diesbezüglich zunächst, dass Art. 11 - zusammen mit Art. 67 - BV das Kindeswohl zur Verfassungsmaxime erhebt, 16 in der sowohl das in Art. 13 Abs. 1 BV enthaltene elterliche Erziehungsrecht wie auch der staatliche Bildungsauftrag gemäss Art. 62 Abs. 2 BV ihre Rechtfertigung und Begrenzung finden. ${ }^{17}$ Während Art. 67 Abs. 1 BV dessen objektivrechtlich-programmatische Dimension kodifiziert (vgl. auch Art. 41 Abs. 1 lit. f und g BV), ${ }^{18}$ handelt es sich bei Art. 11 Abs. 1 BV um die subjektivverfassungsrechtliche Verbürgung des Kindeswohlprinzips. Angestrebt wird mit ihm «eine altersgerechte Entfaltungsmöglichkeit des Kindes in geistigpsychischer, körperlicher und sozialer Hinsicht, wobei in Beachtung aller kon-

15 Insofern ist von den beiden in der Fn. 8 skizzierten Entscheiden (Stichwort: «Yoga im Kindergarten») derjenige des Bundesgerichts eine Spur überzeugender.

16 Vgl. BGE 132 III 359 E. 4.4.2.

17 Reich, «Homeschooling» (Fn. 4), S. 586.

18 Art. 67 Abs. 1 BV ist an sich überflüssig, weil sich sein Gehalt bereits vollständig aus (der programmatischen Schicht von) Art. 11 Abs. 1 BV ergibt. kreten Umstände nach der für das Kind bestmöglichen Lösung zu suchen ist». ${ }^{19}$

14 Des Weiteren ist bedeutsam, dass sich die Tragweite der anspruchsbegründenden Teilgehalte von Art. 11 Abs. 1 BV (und Art. 3 KRK) angesichts von Art. 19 BV und der zahlreichen einfachgesetzlichen Vorgaben, die das Kindeswohlprinzip konkretisieren - zu denen ganz unbestreitbar auch $\S 135$ SchulG/BS gehört (vgl. insbesondere dessen Abs. 2 lit. b) -, rechtspraktisch weitgehend darin erschöpft, im infrakonstitutionellen Bereich bestehende Schutzlücken zu füllen. ${ }^{20}$ Womit wir beim Kern des Problems angelangt wären: Geht man davon aus, dass häuslicher Privatunterricht zu einer Verwirklichung des Kindeswohls führen kann (andernfalls wäre er von vornherein verfassungswidrig), dann darf «Homeschooling» nicht vollständig untersagt sein. ${ }^{21}$ Woraus folgt: Gäbe es

19 BGE 129 III 250 E. 3.4.2 (Hervorhebung hinzugefügt); vgl. ferner Urteil des Bundesgerichts 5A_111/2019 vom 9. Juli 2019, E. 2.3; Cyril Hegnauer, Grundriss des Kindesrechts, 5. Aufl., Bern 1999, N. 26.04a; zum Kindeswohl als einem in erster Linie von den Eltern treuhänderisch zu konkretisierenden, multifunktionalen «Optimierungsgebot» sowie zu den Querbezügen zwischen Art. 11 Abs. 1 BV, Art. 302 ZGB und Art. 3 der UNO-Konvention über die Rechte des Kindes (KRK; SR 0.107) Reich, «Schutz der Kinder und Jugendlichen» (Fn. 9), S. 374 f., 375 ff., 378 f., 383,384 f., der übrigens einen verfassungsrechtlichen Anspruch von Kindern und Jugendlichen befürwortet, im Vorfeld sie betreffender behördlicher «Homeschooling»-Entscheide angehört zu werden (vgl. S. 381, Fn. 161; vgl. auch Reich, «Homeschooling» [Fn. 4], S. 606).

20 Vgl. Reich, "Schutz der Kinder und Jugendlichen» (Fn. 9), S. 381.

21 Weitergehend Reich, «Homeschooling» (Fn. 4), für den es bereits genügt, wenn «Homeschooling» dem Kindeswohl nicht zuwiderläuft: «Für die rechtliche Beurteilung der Zulässigkeit des «Homeschooling» im konkreten Einzelfall ist daher regelmässig die Frage entscheidend, ob sich die normativ vorgegebenen Zwecke des Grundschulunterrichts durch häuslichen Privat- 
in einem Kanton keine gesetzliche Grundlage für die Erteilung des Grundschulunterrichts durch die Eltern, müsste «Homeschooling» dort zumindest insoweit, als es das Kindeswohl in besonders gelagerten Einzelfällen ausnahmsweise ${ }^{22}$ gebietet, dennoch zugelassen werden, und zwar direkt gestützt auf Art.11 Abs. 1 (i. V. m. Art. 13 Abs. 1 und Art. 19) $B V .23$ Solange solche - gleichsam «ein-

unterricht genügend verwirklichen lassen. Ist dies nicht [...] der Fall, kann sich auf dieser Basis ein Eingriff in die elterlichen Erziehungsrechte [...] rechtfertigen» (S. 601; Hervorhebungen i. O.); ganz anders das Bundesgericht, wenn es im Urteil 2C_1005/2018 vom 22. August 2019 heisst, es liege im Ermessen der Kantone, $o b$ «Homeschooling» überhaupt zulässig ist oder nicht (vgl. die E. 5.5). - Zur Vereinbarkeit von «Homeschooling» mit Art. 62 Abs. 2 BV siehe Reich, «Homeschooling» (Fn. 4), S. 592 ff.

$22 \S 135$ Abs. 2 lit. a SchulG/BS verlangt, dass «nachweisbar besondere Gründe vorliegen, dass ein Unterrichtsbesuch nicht möglich ist». Verneint wurde eine «besondere Situation des Kindes» etwa in SOG 2015 Nr. 29, E. 7, weil es «namentlich nicht behindert» war.

23 Ein verfassungsmässiges Recht auf «Homeschooling» befürworten - neben Reich - Eylem Copur/Tarek Naguib, in: Tarek Naguib/Kurt Pärli/ Eylem Copur/Melanie Studer (Hrsg.), Diskriminierungsrecht, Bern 2014, N. 277, 286, «wenn eine angemessene oder eine Idealbeschulung derzeit nicht gewährleistet ist und sich Eltern [sic] damit nicht für [sic] einverstanden erklären möchten». - Zur Relevanz von Art. 19 BV hinsichtlich der Bewilligungsfähigkeit privaten Heimunterrichts Urteil des Bundesgerichts 2C_686/2011 vom 25. Januar 2012, E. 2.3.3 f., wo es ebenfalls um $\S 135$ SchulG/BS ging, der damals allerdings noch weniger streng formuliert war; vgl. in diesem Zusammenhang überdies Regula Kägi-Diener, in: St. Galler Kommentar BV (Fn. 3), Art. 19 N. 17-19, 23. Ein Recht auf «Homeschooling» gibt es im kantonalzürcherischen Verfassungsrecht (vgl. Art. $15 \mathrm{KV} / \mathrm{ZH}$; dazu Urteil des Bundesgerichts 2C_592/2010 vom 20. September 2011, E. 3.2), nicht jedoch im basel-städtischen (vgl. Urteil des Appellationsgerichts BS [als Verwaltungsgericht] VD.2018.97 vom 25. September 2018, E. 2.2; bestätigt in Urteil des Bundesgerichts 2C_1005/2018 vom 22. August 2019, E. 3) oder st. gallischen (vgl. Urteil des Bundesgerichts 2C_738/2010 vom 24. Mai 2011, E. 3.4). schränkend-grundrechtskonkretisierenden» - Normen wie $\S 135$ SchulG/BS bestehen, ${ }^{24}$ wird diese verfassungsrechtliche Minimalgarantie aber allemal auf Gesetzesstufe «konsumiert».

Zusammenfassend: Ein (kantonal-)gesetzliches Verbot häuslichen Privatunterrichts wäre mit Art. 11 BV genauso unvereinbar wie dessen voraussetzungslose Zulässigkeit. Wer einen (bundes-) verfassungsmässigen Individualanspruch der Kinder und Jugendlichen auf «Homeschooling» hingegen kategorisch verneint, bringt damit indirekt nichts anderes zum Ausdruck, als dass es für ein Kind unter keinerlei Umständen auch nur vorübergehend besser sein kann, zuhause beschult zu werden. Und das ginge dann wohl doch etwas zu weit. Es bleibt zu hoffen, dass die Kantone ihre jeweiligen «Homeschooling»-Regelungen grundrechtskonform handhaben und dass das Bundesgericht Art. $11 \mathrm{BV}$ (nicht nur) in künftigen die Zulässigkeit privaten Heimunterrichts betreffenden Fällen mehr Beachtung schenkt.

24 Einzelne Kantone sehen für «Homeschooling» keine Bewilligungs-, sondern bloss eine Meldepflicht vor (vgl. die $\S \S 4$ Abs. 4 und 58 Abs. 3 SchulG/AG und § 34 VolksschulV/AG sowie $\S 69$ Abs. 2 VSG/ZH und § 73 Abs. 1 VSV/ZH); gewisse verzichten auf das Erfordernis eines Lehrdiploms (so der Kanton Bern, wo ein Lehrdiplom ganz generell nicht zwingend ist; vgl. Art. 71a Abs. 1 VSG/BE und Art. 5 LAG/BE). 